\title{
Workflow for a Timber Joinery Robotics
}

\author{
Francisco Javier Quitral-Zapata \\ Universidad Técnica Federico Santa María | Chile | francisco.quitral@usm.cl \\ Luis Felipe González-Böhme \\ Universidad Técnica Federico Santa María | Chile | luisfelipe.gonzalez@usm.cl \\ Rodrigo García-Alvarado \\ Universidad del Bío-Bío | Chile | rgarcia@ubiobio.cl
}

Alejandro Martínez-Rocamora

Universidad de Sevilla | España | rocamora@us.es

\begin{abstract}
We present a novel workflow for timber joinery robotics in low-rise building construction. A parametric $3 \mathrm{D}$ model that associates architectural design, structure geometry and robotic fabrication information was implemented using only CAD-based visual robot programming. Our case study is the design and manufacturing process of a two-story timber-framed dwelling. The main frames of the structure were assembled with mortise and tenon timber joints machined in glue-laminated timber using a 7-axis industrial robot in a wood company. This pioneering experience aims to apply timber framing robotics to social housing in emerging countries.
\end{abstract}

Keywords: Robots in architecture; Robotic timber construction; Timber framing; Timber Joinery Robotics; Visual robot programming.

\section{INTRODUCCIÓN}

El hormigón y el acero son los materiales predominantes en la industria de la construcción. Éstos dejan una gran huella de carbono y requieren grandes cantidades de energía para ser producidos. La madera, en cambio, es un recurso natural renovable que tiene la propiedad inherente de almacenar grandes cantidades de carbono. Como material de construcción, su uso ayuda a reducir el CO2 de la atmósfera, esto la convierte en una alternativa ideal para reducir emisiones y almacenar carbono en nuestros edificios (Green, 2012). Esto ha contribuido a fomentar su uso en la industria de la construcción en un clima social sensible al cuidado del medio ambiente y a la mitigación del cambio climático. La ciencia también ha contribuido a esta percepción mediante la investigación y desarrollo de nuevos productos que permiten soslayar la susceptibilidad de la madera a la humedad o a la pudrición, e incluso incrementar su resistencia al fuego. La madera como materia prima se procesa fácilmente, se puede trabajar a mano o con máquinas-herramienta, en taller o en obra, de manera artesanal o industrializada en grandes líneas de producción. En relación con su volumen, es un material liviano, lo que permite un fácil transporte y manejo. Esta versatilidad la distingue de otros materiales de construcción que requieren procesos, herramientas o entornos más especializados. La reaparición a nivel mundial de la madera como material de construcción incluso para edificación de gran altura, se debe principalmente a la I+D de la madera de ingeniería. El incremento de su resistencia estructural, de su estabilidad dimensional y precisión han permitido disminuir el margen de error típico atribuible a la construcción con madera aserrada.

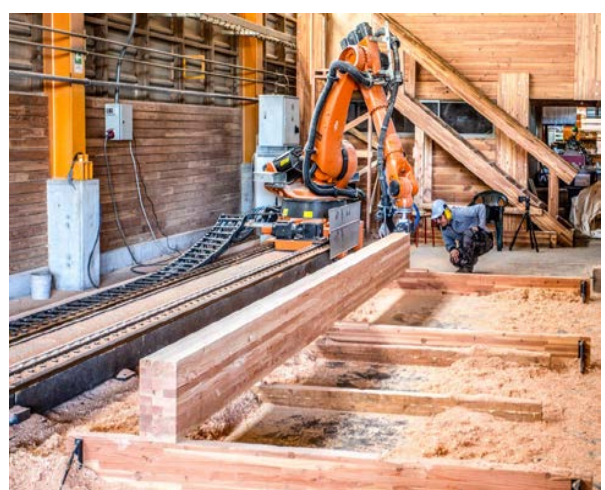

Figura 1: Celda de trabajo, robot KUKA KR240-2 con unidad lineal en aserradero Voipir (Villarrica, Chile).

Aunque existe consenso en que la construcción en madera es una manera efectiva de combatir el cambio climático desde el sector de la construcción (FAO/UN, 2016), en Chile todavía representa apenas el 18\% de la edificación, mientras en otros países con la misma vocación forestal esta cuota sobrepasa el $80 \%$ (Zilic et al., 2019). Por otro lado, la implementación de tecnologías de manufactura avanzada en la construcción ha sido visualizada como una gran oportunidad para incrementar la productividad del sector que ha estado disminuyendo a nivel mundial (Bock, 2015). La construcción robotizada es también una gran alternativa para mejorar el desempeño de la construcción en madera. Sin embargo, la industrialización en la construcción en Chile es todavía muy incipiente, especialmente usando la madera como material predominante. Para una construcción en madera 

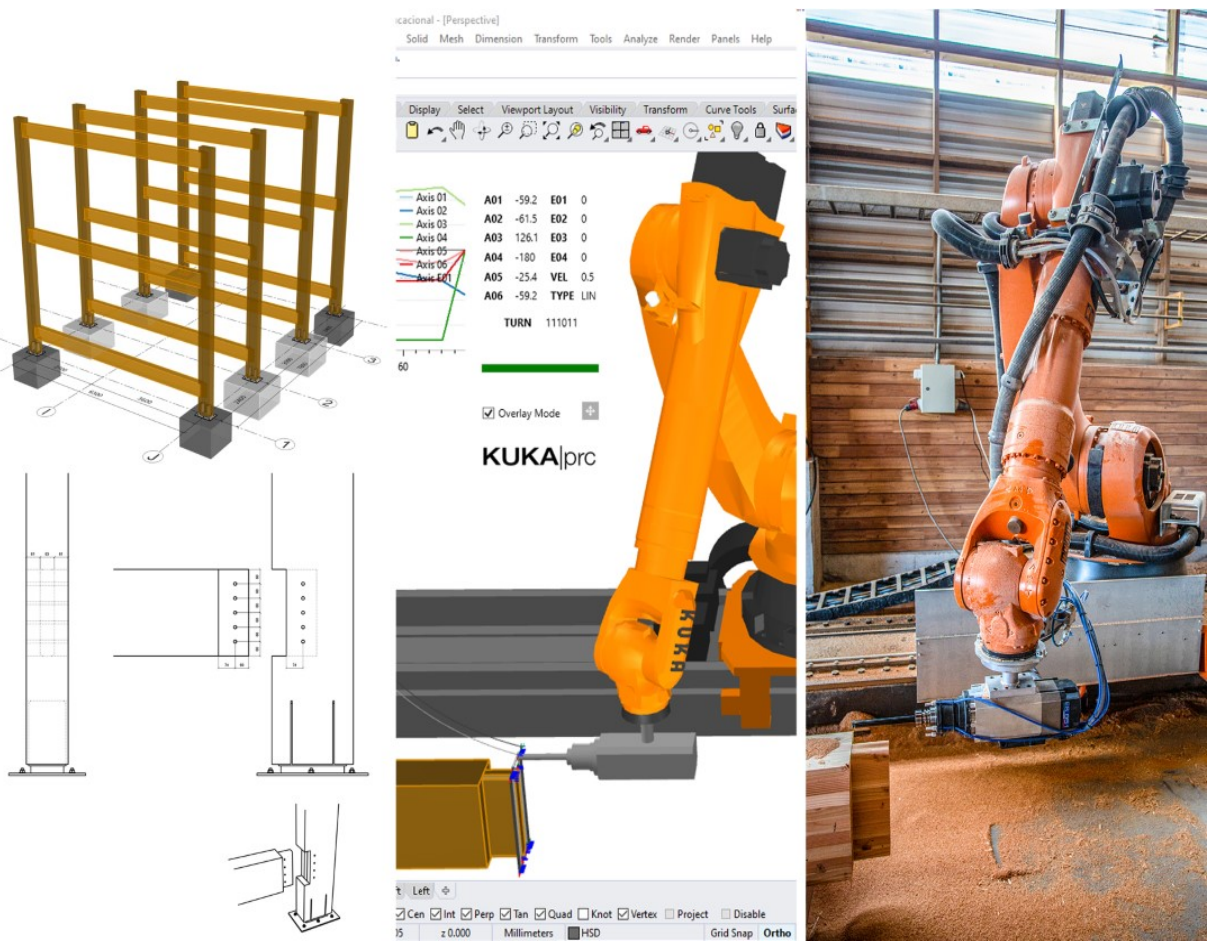

15 20.000 Malimeters 口HSO Gidsop or to

Figura 2: Diseño-Programación-Ejecución de la Carpintería de Armar Robotizada.

industrializada es relevante la demanda de conocimiento de diseñador experto en aplicaciones CAD/CAM/CNC, para desarrollar el diseño detallado en madera de los proyectos. A esto se debe sumar la demanda de mano de obra calificada para operar y mantener la maquinaria CNC. Tiempo y costo de formación de una fuerza de trabajo que satisfaga estos requerimientos podrían ser muy superiores a los necesarios para investigar y desarrollar nuevos flujos de trabajo que permitan habilitar una construcción robotizada en madera.

El propósito de este trabajo es validar empíricamente un flujo de trabajo asociativo desde el diseño arquitectónico y estructural hasta la información de fabricación robotizada y su implementación en un entorno industrial. Se desarrolló una estrategia de modelación $3 \mathrm{D}$ paramétrica para el mecanizado robótico de uniones carpinteras. Se diseñó y fabricó una estructura de poste y viga, armada con ensambles de caja y espiga. Dicha estructura albergará un prototipo de unidad de vivienda de dos niveles. Para su ejecución, un robot industrial mecanizó postes y vigas de madera laminada. Esta experiencia pionera se llevó a cabo en el aserradero Voipir en Villarrica, Chile (Figura 1).

\section{CARPINTERÍA DE ARMAR ROBOTIZADA (CAR)}

En ingeniería de la madera, se pueden distinguir tres tecnologías para conectar piezas estructurales: uniones mecánicas, uniones adhesivas y uniones carpinteras. Las uniones mecánicas utilizan clavos, tornillos, pernos y/o conectores metálicos; estos productos representan el método de conexión más popular en la industria de la construcción en madera ya que existen estándares modernos de diseño de uniones mecánicas muy detallados (European Committee for Standardization, 2014). Las uniones adhesivas permiten cohesionar piezas mediante un enlace superficial; utilizadas comúnmente en la denominada madera masiva (mass timber) como la madera laminada (Glue-laminated timber) o contralaminada (Cross-laminated timber), permiten conformar piezas estructurales eficientes y duraderas. Las uniones carpinteras representan el método tradicional para armar estructuras de madera; muy populares hasta mediados del siglo XIX, se diseñaban y fabricaban tradicionalmente en función de la experiencia y habilidad de los carpinteros de armar de la época. La pérdida de este conocimiento ancestral, los altos costos de mano de obra calificada para la carpintería de armar y nuevos enfoques de diseño redujeron la competitividad de las uniones carpinteras en la industria de la construcción en madera.

La Carpintería de Armar es un método ancestral de construcción sustentable (Gaztelu, 1899) que involucra un sistema constructivo basado en la prefabricación de piezas lineales de madera que se encajan y se traban mediante ensambles y empalmes sin aporte de metal para formar un entramado continuo resistente. Cuenta con la particularidad de que dichas piezas pueden ser desmontadas individualmente para ser reparadas o reemplazadas, sin alterar la integridad de una estructura en pie. Un gran número de tratados y manuales de carpintería fueron publicados en siglos pasados, sin embargo, en los últimos 30 años se han publicado 3 libros relevantes en los que se describen con detalle los parámetros geométricos de una cantidad razonable de uniones carpinteras: Sumiyoshi \& Matsui (1991), Gerner (1992) y González (2019). La adopción de tecnología CNC 
en talleres de carpintería durante los 1990s condujo hacia el redescubrimiento de las uniones carpinteras, atrayendo nuevamente parte de la atención del mundo científico hacia la Carpintería de Armar (Schindler, 2009). Hoy, en los albores de la Industria 4.0, poco se ha investigado aún para desarrollar una Carpintería de Armar Robotizada (González, Quitral, et al., 2017).

\section{PROGRAMACIÓN DE ROBOTS INDUSTRIALES}

Para la prefabricación en serie de componentes constructivos en madera se requiere una superficie extensa para instalar maquinaria y software CAD/CAM/CNC como Cadwork, Sema, Dietrich's o Hsbcad y hardware como las ensambladoras CNC Hundegger, Weinmann o Krüsi, que son herramientas dedicadas, es decir, de propósito específico. Además, la madera a mecanizar idealmente debe ser industrializada como la laminada o la contrachapada, porque ofrece mayor estabilidad dimensional y menor deformación que la madera aserrada. En general, la baja calidad de la madera aserrada es un obstáculo para asegurar que los componentes prefabricados en taller puedan ser montados en obra sin problemas.

La principal ventaja para el sector de la manufactura en madera es que los robots industriales (RIs) son manipuladores multipropósito (International Organization for Standardization, 2012), con un alto potencial de flexibilidad inherente, que las máquinas-herramienta CNC no tienen en igual medida, ni mucho menos las ensambladoras CNC. La manufactura flexible permite fabricar una amplia variedad de productos en lotes pequeños y plazo de entrega mínimo, así como reducir los inventarios. La principal desventaja para la aplicación de RIs en manufactura es que toma más tiempo programar un $\mathrm{RI}$, que una máquina herramienta CNC y mucho más tiempo que una ensambladora CNC. Según Thomessen et al. (2004), puede demorar hasta 400 veces más tiempo programar que ejecutar una amoladura con un RI. La programación offline de Rls es igualmente difícil, porque se basa en configuraciones de sistema muy precisas y el entorno virtual de programación/simulación debe ser calibrado cuidadosamente, para capturar con detalle los parámetros del entorno físico de operación y evitar reprogramar el robot una vez iniciada la producción. La programación online puede evadir este problema, pero en el entretanto, el manipulador no puede seguir produciendo. Los dos métodos disponibles para la programación online de RIs, Teach-Pendant y Lead-Through, son exhaustivos y laboriosos. Si bien el primero permite un posicionamiento en coordenadas $\mathrm{XYZ}$ más preciso del punto central de la herramienta montada en la brida del manipulador, requiere conocimiento de programador experto y es mucho más lento de aplicar que el segundo. En robots colaborativos o "Cobots", el método Lead-Through es auxiliado por los sensores de torque instalados de fábrica. Por otra parte, la programación offline actualmente permite usar diversos entornos de programación basados en CAD/CAM, incluso en combinación con entornos de diseño paramétrico $A A D$ (Algorithm Aided Design). En la industria creativa destacan desde hace algún tiempo los entornos gráficos de programación/simulación KUKA|prc (Braumann \& BrellCokcan, 2011) y HAL (Schwartz, 2013), que permiten parametrizar la trayectoria de Rls y Cobots para fines diversos. Esta clase de aplicaciones habilitan la integración de diseño variacional en la manufactura avanzada asistida por Rls. Resultados previos (González, Maino, et al., 2017) validaron el uso de KUKA|prc para obtener un mecanizado asociativo que actualiza de manera automática el número, distanciamiento o profundidad de pasadas respecto de los cambios de geometría del modelo de la pieza o de la herramienta. Actualmente no existen métodos específicos de programación de Rls que consideren los cambios de diseño en obra -una práctica bastante frecuente en las obras de construcción convencionales.

\section{FLUJO DE TRABAJO CAR}

Un primer objetivo de esta investigación consistió en definir un conjunto de herramientas computacionales para la asociatividad del diseño arquitectónico y estructural; la programación para el mecanizado de uniones carpinteras y la información de la celda de trabajo robotizada (Figura 2). El modelado CAD fue desarrollado en Rhinoceros $®$; la modelación $3 D$ paramétrica de la estructura y de los ensambles fue desarrollada en el entorno de programación visual Grasshopper® y se utilizó KUKA|prc para la simulación de las trayectorias de mecanizado robótico de las uniones carpinteras.

A partir del diseño arquitectónico del prototipo de unidad de vivienda de dos niveles y de sus crujías estructurales se realizó la modelación 3D paramétrica global de la estructura de poste y viga, asociando parámetros descriptivos de la geometría local de los ensambles de caja y espiga. Una vez coordinadas las especialidades de arquitectura y el cálculo estructural, se realizaron pruebas con el RI para precisar y corregir a pie de máquina las tolerancias y holguras producto del fresado sobre las piezas de trabajo (Figura 3). De este modo, se actualizaron los valores nominales del modelo $3 \mathrm{D}$ paramétrico de las uniones carpinteras, sus trayectorias de mecanizado y las discrepancias en la información espacial de la celda de trabajo robotizada.

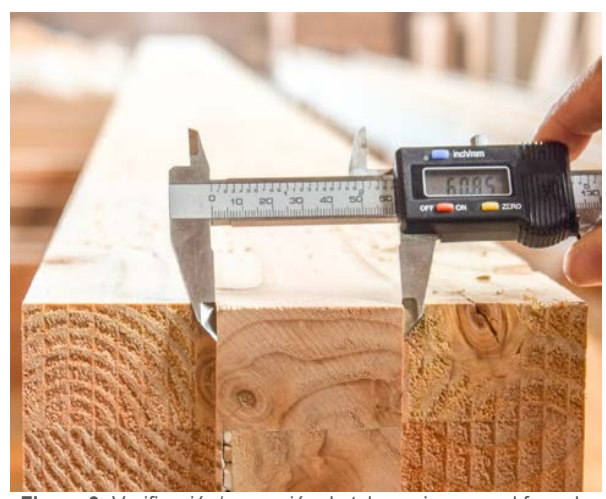

Figura 3: Verificación/corrección de tolerancias para el fresado de una espiga en viga de madera laminada.

Posteriormente, el robot industrial mecanizó 8 postes (de $185 \times 360 \times 6600 \mathrm{~mm}$ ) y 12 vigas (de $185 \times 360 \times 6300 \mathrm{~mm}$ ) en madera laminada de pino Oregon (pseudotsuga menziesii). El mecanizado se ejecutó en una celda de trabajo compuesta por un robot industrial KUKA KR240-2, montado sobre una unidad lineal, equipado con un electrohusillo HSD ES951 y una fresa recta (con cuchillas intercambiables) de $200 \mathrm{~mm}$ de largo útil y $25 \mathrm{~mm}$ de diámetro. 


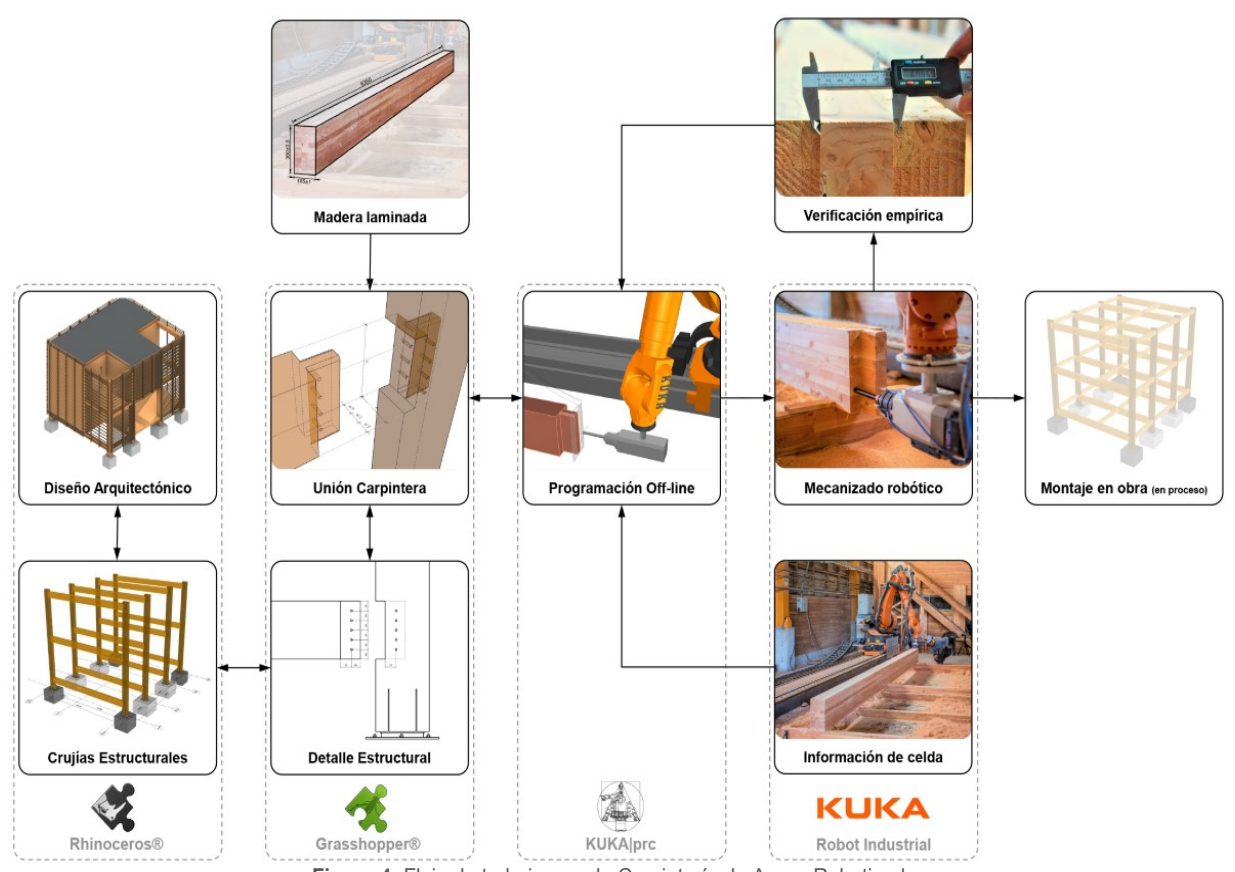

Figura 4: Flujo de trabajo para la Carpintería de Armar Robotizada.

El grupo de investigación en Carpintería de Armar Robotizada de la Universidad Técnica Federico Santa María, en Valparaíso, llevó a cabo la modelación paramétrica y la programación/simulación de trayectorias de mecanizado de manera remota y asincrónica respecto de la manufactura en la celda de trabajo robotizada, ubicada en Villarrica, a $870 \mathrm{~km}$ de distancia. Esta restricción geográfica imponía un requerimiento relevante para la definición de un flujo de trabajo asociativo (Figura 4) que permitiera actualizaciones automáticas de la información para la manufactura cuando surgieran nuevas condiciones o valores emergentes de diseño.

\section{RESULTADOS}

Esta investigación permitió desarrollar un flujo de trabajo asociativo desde el diseño arquitectónico y estructural hasta la información de fabricación robotizada y su implementación en un entorno industrial. Con el flujo de trabajo CAR se logró simular/ejecutar trayectorias de mecanizado, verificar y corregir a pie de máquina las tolerancias entre los modelos 3D nominales y las piezas de trabajo mecanizadas. Durante las pruebas de fresado se determinaron un conjunto de parámetros que permitieron obtener rápidamente resultados aceptables; profundidad de corte (cut depth o stepdown) de $2 \mathrm{~mm}$; ancho de corte (cut width o stepover) de $8 \mathrm{~mm}$; velocidad del electrohusillo (spindle speed) de 12.000 revoluciones por minuto r/min; velocidad de avance para el desbaste (roughing feed rate) $0,15 \mathrm{~m} / \mathrm{s}$; velocidad de avance para el acabado (finishing feed rate) $0,05 \mathrm{~m} / \mathrm{s}$. Estos valores permitieron una relación tiempo/calidad de mecanizado admisible.

En total, se mecanizaron 24 ensambles de caja y espiga; con 2 postes y 3 vigas se conforma cada uno de los 4 marcos de la estructura prototipo (Figura 2). El tiempo de mecanizado efectivo de cada caja fue de 1,34 horas; se ejecutaron 3 cajas por cada poste $(\times 8)$, lo que da un total de 32,16 horas. El tiempo de mecanizado efectivo de cada espiga fue de 1,4 horas; se ejecutaron 2 espigas por cada viga $(\times 12)$, lo que da un total de 33,6 horas. En un tiempo total aproximado de 66 horas se ejecutaron los 24 ensambles. No se cronometraron las pruebas de mecanizado ni los tiempos de carga y descarga de las piezas de trabajo. Cabe señalar que los parámetros de fresado se podrían optimizar para reducir los tiempos de mecanizado, sin embargo, esto no era un objetivo perseguido en la investigación.

Por cronograma, primero se mecanizaron las espigas de las vigas (Figura 5), esto permitió verificar las tolerancias dimensionales de 24 instancias de mecanizado. Este conjunto de datos permitió actualizar los valores geométricos de las cajas y sus trayectorias de mecanizado en el modelo 3D paramétrico. De este modo, fue posible incluir holguras para las instancias de mecanizado de las cajas. Se ejecutaron holguras $\leq 2 \mathrm{~mm}$ en el alto; $\leq 1,5 \mathrm{~mm}$ en el ancho y de $\leq 2 \mathrm{~mm}$ en la profundidad de las cajas, para facilitar luego el montaje de las espigas.

Se detectaron algunas inestabilidades connaturales de la madera, deformaciones por el peso propio, errores en el posicionamiento de las piezas de trabajo tras operaciones de carga/descarga, sin embargo, todas estas desviaciones no fueron incluidas para esta investigación.

\section{DISCUSIÓN}

En Chile, incluso la madera de ingeniería tiene una gran tolerancia dimensional; $+/-2 \mathrm{~mm}$ en el ancho, $+1 \%$ con un máximo de $3 \mathrm{~mm}$, cada $300 \mathrm{~mm}$ en la altura, admisibles por norma (Instituto Nacional de Normalización, 2013), sin considerar deformaciones por el peso propio. En la industria de la construcción, la madera como material es 
producido y ensayado con un bajo contenido de humedad, debido a sus propiedas higroscópicas, los cambios de temperatura y de humedad relativa del ambiente también producen cambios dimensionales y deformaciones en la madera. Estas tolerancias impiden que los procesos robóticos de manipulación y posicionamiento de miembros de madera sean lo suficientemente precisos. Es más, se generan tolerancias por acumulación que causan problemas importantes durante el proceso de montaje (Eversmann et al., 2017; Willmann et al., 2016). De manera experimental se reporta que para el mecanizado con un RI se debe considerar una tolerancia entre 0,5 a $1 \mathrm{~mm}$ para que un ensamble pueda encajar (Heesterman \& Sweet, 2018). Sin embargo, esta no es una regla que se pueda generalizar, pues, cada relación entre miembros de una unión necesita ser probada físicamente para determinar la tolerancia ideal.

En un modelo CAD tridimensional, la representación idealizada de un miembro lineal de madera, es suficiente para realizar simulaciones estructurales (Kohlhammer et al., 2017) pero para muchos procesos sustractivos de piezas de geometría compleja, aparecen problemas adicionales cuando se trata del montaje de materiales que tienen tolerancias muy altas (Stumm et al., 2017). Estas tolerancias o errores, independiente de su origen, se acumulan durante las diversas fases del proyecto, desde la fabricación hasta el montaje de componentes (Brugnaro et al., 2019). Muchos de estos errores se han resuelto en el estado del arte mediante la implementación de mecanismos de retroalimentación basados en sensores que permiten absorber las incongruencias entre la realidad física y los modelos digitales. En investigaciones recientes, una posible estrategia consiste en establecer un enfoque en la precisión local de la unión en lugar de la precisión global del sistema (Thoma et al., 2018). Otra estrategia posible consiste en la colaboración humano-máquina como forma efectiva de manejar las tolerancias (Adel et al., 2018).

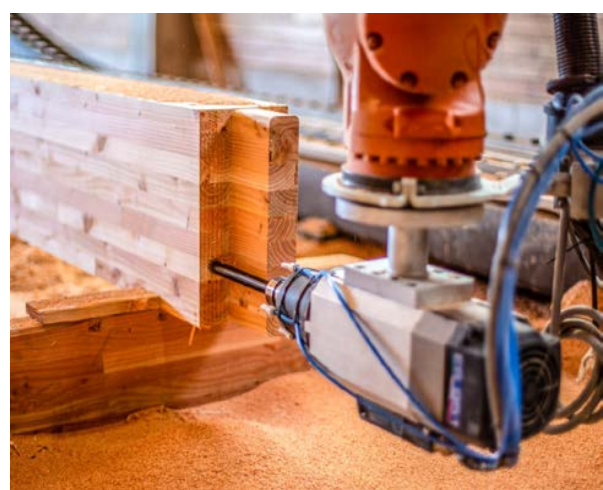

Figura 5: Mecanizado de espiga en viga de madera laminada de pino Oregon.

\section{CONCLUSIONES}

El flujo de trabajo desarrollado en esta investigación y validado a nivel industrial ofrece varias ventajas competitivas como, por ejemplo:

- Simular trayectorias de mecanizado asociativas, que se actualizan automáticamente cuando el usuario asigna nuevos valores; de las crujías o escuadrías, de los parámetros geométricos de las uniones carpinteras, de la celda de trabajo del robot o de la herramienta, etc.

- Reducir errores de manufactura con la posibilidad de incorporar correcciones durante la producción.

- Asegurar repetibilidad en la producción con la posibilidad de retroalimentar tolerancias entre los modelos nominales y las piezas de trabajo.

- Contar con una mayor variabilidad en el diseño arquitectónico y adaptabilidad a los cambios de diseño durante las distintas etapas del proyecto.

- Aumentar la reciprocidad entre el diseño arquitectónico/estructural y la información para la manufactura.

- Automatizar el mecanizado de uniones carpinteras habilitando su uso estructural para la construción de vivienda.

- Ampliar el catálogo de productos y servicios habilitando una manufactura flexible de lotes pequeños reduciendo plazos de entrega.

Una desventaja aparente del flujo de trabajo CAR es la necesidad de implementación ad hoc para la configuración específica de la celda de trabajo robotizada; sin embargo, una ampliación el catálogo de productos hacia una verdadera manufactura flexible permitiría alcanzar una buena relación costo-beneficio para la industria.

Rhinoceros ${ }^{\circledR}$, Grasshopper ${ }^{\circledR}$ y KUKA|prc habilitan la integración de diseño variacional en la manufactura avanzada asistida por robots industriales. La posibilidad de integrar información geométrica de la materia prima, de los procesos 0 de los sistemas abre posibilidades completamente nuevas para la industria de la construcción en madera. La investigación y desarrollo en Carpintería de Armar Robotizada es aún incipiente y presenta muchos desafíos teóricos, prácticos y metodológicos. En el caso específico de uniones carpinteras, se requiere de una pequeña holgura para que los ensambles sean útiles, es decir, un pequeño espacio entre las dos piezas que han de encajar entre sí. Estas uniones no deben quedar demasiado flojas, lo que disminuye la rigidez estructural y estética de un entramado, ni demasiado apretadas, lo que impide o dificulta el montaje. Para evitar errores de manufactura aislados que afectan de manera sistemática a un conjunto de piezas de un entramado de madera, se requieren procesos lo suficientemente flexibles para operar con los efectos de los materiales, que no pueden ser modelados de manera nativa en las herramientas CAD/CAM convencionales. Esta conclusión sugiere orientar nuestro trabajo futuro hacia nuevas herramientas/flujos de trabajo con mayor integración entre sistemas, nuevos canales de comunicación que habiliten una operación colaborativa entre humanos y robots para aprovechar la sinergia de sus capacidades.

\section{AGRADECIMIENTOS}

Este trabajo fue financiado por el U.S. Department of Energy Solar Decathlon Build Challenge 2020 y la Universidad Técnica Federico Santa María (UTFSM) en el contexto del desarrollo del prototipo "Casa FENIX 2.0" del Departamento de Arquitectura. Para obtener más información, por favor visite: arquitectura.usm.cl/; vimeo.com/archcomputingutfsm 
La investigación fue financiada por la Agencia Nacional de Investigación y Desarrollo / Subdirección de Capital Humano / Beca de Doctorado Nacional - Año Académico 2020 - 21202373 y el programa de Doctorado en Arquitectura y Urbanismo de la Universidad del Bío-Bío. Un agradecimiento especial a Miguel González del aserradero Voipir.

\section{REFERENCIAS}

Adel, A., Thoma, A., Helmreich, M., Gramazio, F., \& Kohler, M. (2018). Design of robotically fabricated timber frame structures. Recalibration on Imprecision and Infidelity Proceedings of the 38th Annual Conference of the Association for Computer Aided Design in Architecture, ACADIA 2018, 394-403.

Bock, T. (2015). The future of construction automation: Technological disruption and the upcoming ubiquity of robotics. Automation in Construction, 59, 113-121. https://doi.org/10.1016/j.autcon.2015.07.022

Braumann, J., \& Brell-Cokcan, S. (2011). Parametric robot control: Integrated CAD/CAM for architectural design. Integration Through Computation - Proceedings of the 31st Annual Conference of the Association for Computer Aided Design in Architecture, ACADIA 2011, 242-251.

Brugnaro, G., Figliola, A., \& Dubor, A. (2019). Negotiated Materialization: Design Approaches Integrating Wood Heterogeneity Through Advanced Robotic Fabrication. In F. Bianconi \& M. Filippucci (Eds.), Digital Wood Design: Innovative Techniques of Representation in Architectural Design (Vol. 24, pp. 135-158). Springer International Publishing. https://doi.org/10.1007/978-3-030-03676-8_4

European Committee for Standardization. (2014). EN 1995-11:2004+A2:2014 Eurocode 5: Design of timber structures Part 1-1: General - Common rules and rules for buildings. British Standards Institution.

Eversmann, P., Gramazio, F., \& Kohler, M. (2017). Robotic prefabrication of timber structures: towards automated large-scale spatial assembly. Construction Robotics, 1(14), 49-60. https://doi.org/10.1007/s41693-017-0006-2

FAO/UN. (2016). Forestry for a low-carbon future: Integrating forests and wood products in climate change strategies. FAO Forestry Paper 177.

Gaztelu, L. (1899). Carpintería de armar (2nd ed.). Bailly-Baillière e Hijos.

Gerner, M. (1992). Handwerkliche Holzverbindungen der Zimmerer (Deutsche Verlags-Anstalt DVA (ed.)).

González, L. F., \& Maino, S. (2019). Uniones carpinteras de Valparaíso: la geometría de ensambles y empalmes (RIL (ed.)).

González, L. F., Maino, S., Quitral, F., \& Hurtado, M. (2017). Reconstrucción robotizada del patrimonio arquitectónico chileno en madera. SIGraDi 2017 [Proceedings of the 21th Conference of the Iberoamerican Society of Digital Graphics], 236-241. https://doi.org/10.5151/sigradi2017038

González, L. F., Quitral, F., \& Maino, S. (2017). Roboticus tignarius: robotic reproduction of traditional timber joints for the reconstruction of the architectural heritage of Valparaíso. Construction Robotics, 1(1-4), 61-68. https://doi.org/10.1007/s41693-017-0002-6

Green, M. (2012). The case for tall wood buildings: How mass timber offers a safe, economical, and environmental friendly alternative for tall building structures.

Heesterman, M., \& Sweet, K. (2018). Robotic Connections: Customisable Joints for Timber Construction. SIGraDi 2018
[Proceedings of the 22nd Conference of the Iberoamerican Society of Digital Graphics], 644-652. https://doi.org/10.5151/sigradi2018-1358

Instituto Nacional de Normalización. (2013). NCh2148:2013 Madera laminada encolada estructural - Requisitos, métodos de muestreo e inspección. http://tipbook.iapp.cl/ak/7ba2f4bd8e4ba3715cad4afabda5 061914006c38/embed/view/2148\#page/1

International Organization for Standardization. (2012). ISO 8373:2012 Robots and robotic devices - Vocabulary. British Standards Institution.

Kohlhammer, T., Apolinarska, A. A., Gramazio, F., \& Kohler, M. (2017). Design and structural analysis of complex timber structures with glued T-joint connections for robotic assembly. International Journal of Space Structures, 32(34), 199-215. https://doi.org/10.1177/0266351117746268

Schindler, C. (2009). Ein architektonisches Periodisierungsmodell anhand fertigungstechnischer Kriterien, dargestellt am Beispiel des Holzbaus [ETH Zürich]. https://doi.org/10.3929/ethz-a-010782581

Schwartz, T. (2013). HAL: Extension of a visual programming language to support teaching and research on robotics applied to construction. In S. Brell-Çokcan \& J. Braumann (Eds.), Rob | Arch 2012 (pp. 92-101). Springer Vienna.

Stumm, S., Braumann, J., von Hilchen, M., \& Brell-Cokcan, S. (2017). On-Site Robotic Construction Assistance for Assembly Using A-Priori Knowledge and Human-Robot Collaboration. In A. Rodić \& T. Borangiu (Eds.), Advances in Robot Design and Intelligent Control (pp. 583-592). Springer International Publishing.

Sumiyoshi, T., \& Matsui, G. (1991). Wood joints in classical Japanese Architecture (p. 124).

Thoma, A., Adel, A., Helmreich, M., Wehrle, T., Gramazio, F., \& Kohler, M. (2018). Robotic Fabrication of Bespoke Timber Frame Modules. In Robotic Fabrication in Architecture, Art and Design 2018 (pp. 447-458). Springer International Publishing. https://doi.org/10.1007/978-3-319-92294-2_34

Thomessen, T., Sannæs, P. K., \& Lien, T. K. (2004). Intuitive robot programming. Proc. 35th International Symposium on Robotics.

Willmann, J., Knauss, M., Bonwetsch, T., Apolinarska, A. A Gramazio, F., \& Kohler, M. (2016). Robotic timber construction - Expanding additive fabrication to new dimensions. Automation in Construction, 61, 16-23. https://doi.org/10.1016/j.autcon.2015.09.011

Zilic, F., Elissetche, J. P., \& Hernandez, V. (2019). Oportunidades de Manufactura Avanzada para la Industria de la Construcción En Madera. www.polomadera.cl 